\title{
Zika como causa de aborto espontáneo en zonas endémicas
}

\author{
Paola G. Rivadeneyra-Espinar ${ }^{1}$, German A. Venegas-Esquivel ${ }^{1}$, Claudia M. Díaz-Espinoza², \\ Víctor M. Pérez-Robles ${ }^{3 *}$, Mariana I. González-Fernández ${ }^{4}$ y Eduardo Sesma-Medrano ${ }^{5}$ \\ ${ }^{1}$ Departamento de Infectología, Hospital Infantil de México Federico Gómez, Ciudad de México; ${ }^{2}$ Departamento de Infectología, Centro Médico \\ Nacional 20 de Noviembre, Instituto de Seguridad y Servicios Sociales de los Trabajadores del Estado, Ciudad de México; ${ }^{3}$ Dirección de Atención \\ Médica, Servicios de Salud de Morelos, Cuernavaca, Morelos; ${ }^{4}$ Centro Regional de Control de Vectores Oaxtepec, Servicios de Salud de Morelos, \\ Cuernavaca, Morelos; ${ }^{5}$ Coordinación Estatal de Vectores y Zoonosis, Servicios de Salud de Morelos, Cuernavaca, Morelos. México
}

\section{Resumen}

Introducción: Aunque en la mayoría de los casos la infección por el virus del Zika (ZIKV) causa enfermedad febril sin complicaciones, en mujeres embarazadas es causa reconocida de alteraciones congénitas y muerte fetal. La microcefalia es la característica descrita con mayor frecuencia en el síndrome congénito por este virus. Caso clínico: Paciente de sexo femenino de 23 años de edad que cursaba la novena semana de gestación (SDG) por la fecha de la última menstruación y la 10.3 SDG por ultrasonido. Acudió a los servicios de salud por presentar un cuadro clínico compatible con infección por el ZIKV, la cual se confirmó por estudios de laboratorio. A las 18.6 SDG se documentó feto único sin movimientos, con datos de microcefalia, tórax con área cardiaca sin actividad, placenta corporal anterior con calcificación en el $30 \%$ e imagen probable de lago venoso versus quiste placentario. La paciente ingresó en urgencias por aborto diferido. Se indujo el trabajo de parto y se obtuvo por vía vaginal un producto masculino de $80 \mathrm{~g}$, con perímetro cefálico de $9 \mathrm{~cm}$. El análisis del cordón umbilical por la reacción en cadena de la polimerasa con retrotranscriptasa confirmó el diagnóstico de síndrome congénito por ZIKV. Conclusiones: Durante el embarazo debe sospecharse la infección por ZIKV para realizar un diagnóstico oportuno y ofrecer una atención integral. La pérdida del producto de la concepción en estas pacientes se ha documentado, y se ha encontrado ARN del ZIKV en las biopsias de las vellosidades coriónicas, lo que podría sugerir el aborto espontáneo de manera temprana durante la viremia.

Palabras clave: Virus Zika. Aborto espontáneo. Mosquito género Aedes.

\section{Zika as a cause of spontaneous abortion in endemic areas}

\section{Abstract}

Background: Although in most cases Zika is an uncomplicated febrile disease, in pregnant women is a recognized cause of congenital disorders. Microcephaly is the characteristic most frequently described in the congenital Zika virus syndrome, and the diagnosis requires laboratory confirmation. Case report: A 23-year-old female on the ninth week of pregnancy by date of last menstrual period and 10.3 weeks by ultrasound, attended to Health Services, presenting clinical manifestations of Zika infection, which was later confirmed by laboratory tests. In her 18.6 weeks of pregnancy, a non-mobile single fetus was documented. Additional findings were microcephaly, lack of thorax with cardiac activity, anterior corporal placenta with $30 \%$ calcification, and an image of probable venous lake versus placental cyst. She was admitted to the emergency room

Correspondencia:

*Víctor Manuel Pérez-Robles

E-mail: vmprinfectologia@ hotmail.com
Disponible en internet: 11-06-2019 Bol Med Hosp Infant Mex. 2019;76:193-197

www.bmhim.com 
for late abortion and labor was induced, obtaining an $80 \mathrm{~g}$ male product with head circumference of $9 \mathrm{~cm}$. Reverse transcription polymerase chain reaction analysis on the umbilical cord was positive for Zika virus. Conclusions: Zika infection during pregnancy must be suspected and diagnosed promptly to offer comprehensive care. The loss of conception in these patients has been documented with results of chorionic villus biopsies, finding Zika virus RNA and suggesting spontaneous abortion early during viremia.

Key words: Zika virus. Spontaneous abortion. Mosquito genus Aedes.

\section{Introducción}

La infección por el virus del Zika (ZIKV) es una arbovirosis transmitida al humano por mosquitos del género Aedes. El virus pertenece a la familia Flaviviridae, la misma a la que pertenecen también otros flavivirus, como el virus del dengue, el virus de la fiebre amarilla, virus del Nilo Occidental y el virus de la encefalitis japonesa. Desde su reconocimiento como causa de malformaciones congénitas, se ha verificado la presencia del genoma del ZIKV en muestras de tejidos y sangre en recién nacidos con microcefalia, y se ha confirmado por inmunohistoquímica'.

A la fecha, se ha demostrado que la infección prenatal por ZIKV puede ocurrir en cualquier trimestre del embarazo, e incluso la infección puede seguir su curso de forma asintomática en la mayoría de los casos. Por lo tanto, no es posible confirmar la infección en todas las mujeres afectadas ${ }^{2-5}$. La evidencia disponible ha demostrado la transmisión vertical del ZIKV, y se ha encontrado ARN viral en el tejido de la placenta, líquido amniótico, suero y cerebro de los fetos o recién nacidos de madres sintomáticas 0 asintomáticas ${ }^{5-9}$.

La microcefalia es la característica clínica descrita con mayor frecuencia ante la sospecha de infección congénita por ZIKV. El diagnóstico definitivo de la infección requiere de la confirmación en el laboratorio, porque ninguna característica clínica o radiológica patognomónica distingue consistentemente la infección congénita de otras causas infecciosas, tóxicas o genéticas de la microcefalia. Diversos estudios y series de casos han documentado la presencia de ZIKV en las muestras de líquido amniótico de mujeres embarazadas $^{10}$. Existen definiciones operacionales específicas emitidas por la Organización Mundial de la Salud (OMS) para la vigilancia e identificación de casos de síndrome congénito y aborto o muerte fetal asociada a la infección por ZIKV (Tabla 1) ${ }^{11}$.

En México, el brote de ZIKV inició a finales del 2015; primero se detectó en Chiapas, y posteriormente se expandió a los territorios endémicos por medio del vector Aedes aegypti, ${ }^{12}$. Las entidades federativas con el mayor número de casos autóctonos en mujeres embarazadas desde el 2015 al 23 de julio del 2018 son Yucatán y Veracruz ${ }^{13}$. Este último estado concentró el $25 \%$ de los casos confirmados de síndrome congénito asociado con ZIKV $(n=10 \text { casos) })^{14}$.

En el estado de Morelos, México, se han reportado 504 casos confirmados de enfermedad por ZIKV desde el 2015 hasta la semana epidemiológica 32 de 2018. De ellos, 321 se reportaron en mujeres embarazadas ${ }^{13}$. Al momento, solamente se ha confirmado un caso de síndrome congénito por ZIKVV14, el cual se documenta a continuación. Posteriormente, se discute el abordaje diagnóstico en mujeres embarazadas con alta sospecha de infección por ZIKV.

\section{Caso clínico}

Paciente de sexo femenino de 23 años de edad, originaria de Ayala, Morelos, México, con antecedentes de gesta III, para II, que cursa un embarazo aparentemente normal, de 9 semanas de gestación (SDG) por la fecha de la última menstruación (FUM) y 10.3 SDG por ultrasonido con control prenatal adecuado. La paciente acude a control al primer nivel de atención con cuadro de 4 días de evolución caracterizado por exantema, mialgias, artralgias, dolor retroocular y cefalea. Por definición operacional, se considera caso probable de Zika y se solicita la prueba confirmatoria de laboratorio. Se realizó un análisis por reacción en cadena de la polimerasa con retrotranscriptasa (RT-PCR) en suero, y se confirmó el diagnóstico de infección por ZIKV, por lo que se clasificó como embarazo de alto riesgo y se refirió al tercer nivel de atención para seguimiento.

En la décimo primera SDG se tomó control ultrasonográfico, donde se reportó un producto único, vivo, aparentemente sano, de 12 SDG, $50 \mathrm{~g}$, circunferencia cefálica de $60 \mathrm{~mm}$, longitud femoral $6.5 \mathrm{~mm}$, circunferencia abdominal $4.7 \mathrm{~mm}$. Continuó el control en el tercer nivel con evolución aparentemente normal. A las 18.6 SDG, la paciente acudió a la cita de control, en la cual no se localizó latido cardiaco fetal. Se realizó un ultrasonido que reportó feto único sin movimientos, con fetometría promedio de 16.4 SDG, peso de $168 \mathrm{~g}$, diámetro 
Tabla 1. Definiciones operacionales en infección por el ZIKV

\begin{tabular}{|c|c|}
\hline & Características \\
\hline $\begin{array}{l}\text { - Caso de síndrome congénito probable } \\
\text { de estar asociado a la infección por el } \\
\text { ZIKV } \\
\text { - Caso de síndrome congénito confirmado } \\
\text { de estar asociado a la infección por el } \\
\text { ZIKV }\end{array}$ & $\begin{array}{l}\text { Recién nacido vivo que presente } \\
\text { - microcefalia (medida de la circunferencia craneal occipitofrontal por debajo } \\
\text { de -2 SD } 24 \text { horas después de nacer, según las referencias estandarizadas de } \\
\text { acuerdo con la edad gestacional y el sexo o } \\
\text { - alguna malformación congénita del sistema nervioso central y cuya madre, durante } \\
\text { el embarazo, haya tenido } \\
\text { - antecedente de residencia o viaje a un área con presencia de vectores del ZIKV o } \\
\text { - haya tenido relaciones sexuales sin protección con una pareja con antecedente } \\
\text { de residencia o viaje a un área con presencia de vectores del ZIKV } \\
\text { Recién nacido vivo que cumpla con los criterios de caso de síndrome congénito } \\
\text { sospechoso de estar asociado a la infección por el ZIKV y } \\
\text { - presente alteraciones morfológicas intracraneales diagnosticadas por cualquier } \\
\text { método de imagen, excluidas otras posibles causas conocidas o } \\
\text { - cuya madre haya presentado exantema durante el embarazo } \\
\text { Recién nacido vivo, de cualquier edad gestacional, que cumpla con los criterios de } \\
\text { caso de síndrome congénito sospechoso de estar asociado a la infección por el ZIKV y } \\
\text { en quien se haya confirmado la infección por ZIKV con pruebas de laboratorio, } \\
\text { independientemente de la detección de otros agentes }\end{array}$ \\
\hline $\begin{array}{l}\text { Aborto o muerte fetal asociados a la } \\
\text { infección por el ZIKV } \\
\text { - Aborto o muerte fetal con sospecha de } \\
\text { asociación a la infección por el ZIKV }\end{array}$ & $\begin{array}{l}\text { Producto de un aborto o muerte fetal de una gestante que } \\
\text { - durante el embarazo haya presentado exantema y } \\
\text { - con antecedente de residencia o viaje a un área con presencia de vectores para el ZIKV o } \\
\text { - haya tenido relaciones sexuales sin protección durante el embarazo con una pareja con } \\
\text { antecedente de residencia o viaje a un área con presencia de vectores para el ZIKV } \\
\text { Todo caso sospechoso en el que se confirme la infección por el ZIKV a partir de } \\
\text { muestras de sangre u orina de la gestante o puérpera o de tejidos del producto de } \\
\text { aborto o muerte fetal }\end{array}$ \\
\hline
\end{tabular}

SD: desviación estándar; ZIKV: virus del Zika.

biparietal y perímetro cefálico con 4 semanas menor a la estimada por FUM, con remodelación de hueso occipital y parietal, y tórax sin evidencia de actividad cardiaca, movimientos somáticos o reflejos. En el abdomen se identificó una discreta burbuja en la cámara gástrica. Se observaron cuatro extremidades (torácicas y pélvicas), sin descartar malformación estructural. En la placenta se observó inserción corporal anterior, con calcificación del $30 \%$ de la misma y evidencia de lagos venosos versus quiste placentario de aproximadamente $4 \times 3 \mathrm{~cm}$. Líquido amniótico de $2.1 \mathrm{~cm}$, cuantitativamente normal. Se establecieron los diagnósticos: aborto diferido de segundo trimestre y síndrome congénito por ZIKV.

La paciente ingresó y se le realizó un legrado instrumentado. Se obtuvo un producto de sexo masculino de $80 \mathrm{~g}$, con perímetro cefálico de $9 \mathrm{~cm}$ (valor esperado de 15.5 para el percentil 50$)^{15}$. Se tomó una muestra del cordón umbilical para RT-PCR, que resultó positiva para ARN de ZIKV y negativa para toxoplasmosis, rubéola citomegalovirus, herpes simple y virus de la inmunodeficiencia humana, y se emitió el diagnóstico de síndrome congénito por ZIKV. Finalmente, la paciente fue egresada por mejoría clínica.

\section{Discusión}

La infección por ZIKV es una enfermedad emergente causada por un virus del género Flavivirus. Otras vías de trasmisión menos frecuentes son la transfusión sanguínea, sexual y perinatal. Sin embargo, la fisiopatología en la trasmisión perinatal no es clara, ya que solamente en el $29 \%$ de las pacientes que cursan con ZIKV durante el embarazo se desarrollan malformaciones fetales ${ }^{16-18}$.

Modelos animales en primates han demostrado que la infección con ZIKV durante etapas tempranas del embarazo favorece la viremia materna prolongada, neuropatología fetal y pérdida de los productos. Entre los datos histopatológicos de las lesiones cerebrales se documentan microcalcificaciones, hemorragia, necrosis, vasculitis, gliosis o apoptosis de células neuroprogenitoras, las cuales se traducen en alteraciones en el desarrollo y crecimiento del cerebro ${ }^{19}$. 
El fenotipo característico del síndrome congénito por ZIKV en humanos incluye microcefalia grave, atrofia cortical con calcificaciones subcorticales, cicatrización macular y focal de la retina y artrogriposis. Lo anterior, sumado a una prueba de RT-PCR positiva, confirma el diagnóstico posnatal ${ }^{20}$.

En el caso aquí documentado, el diagnóstico de la infección por ZIKV se realizó de manera precoz a las 11 SDG, sin alteraciones aparentes en el producto, por lo que se continuó con el manejo como embarazo de alto riesgo y se observó una evolución aparentemente normal en el tercer nivel de atención. En la tercera cita de control prenatal, se corroboró la muerte fetal ocurrida aproximadamente en la 16 SDG y la presencia de alteraciones típicas del síndrome congénito por ZIKV. El diagnóstico se realizó basándose en los criterios descrito por la OMS (Tabla 1) ${ }^{11}$, los cuales incluyeron la confirmación de la infección por RT-PCR en tejidos del producto de aborto o muerte fetal.

El seguimiento del embarazo, en especial en mujeres que han sido infectadas por ZIKV, es un reto para los sistemas de salud, ya que requiere de una gran cantidad de recursos que deben estar disponibles y próximos a los pacientes. La disponibilidad de pruebas confirmatorias como RT-PCR o ultrasonido puede permitir la identificación, de manera precoz, de la infección. Sin embargo, esta disponibilidad es todavía una debilidad en nuestro entorno, y puede contribuir a la falta de identificación de los casos, un seguimiento inadecuado, el retraso en medidas paliativas - como la interrupción de la gestación cuando exista recomendación para ello- y la falta de documentación de esta patología como causa de abortos y malformaciones congénitas.

En el caso que se documenta, se contó con la disponibilidad del RT-PCR. Sin embargo, a pesar del estrecho seguimiento, se presentó el síndrome congénito y la muerte del producto.

El acceso a estas herramientas es relevante, ya que sin ellas es sumamente complicado realizar un seguimiento estrecho. Además, se limita la opción de la interrupción del embarazo para las mujeres afectadas en los casos en los que no existe posibilidad de vida del producto, con lo cual se podría evitar el sufrimiento innecesario y la espera de lo inevitable.

Estas situaciones deben ser abordadas mediante una mejor capacitación del personal de salud y la infraestructura de la atención de primer nivel en los cuidados del embarazo. Lo anterior, para evitar que la infección por ZIKV se convierta, en el mediano plazo, en una enfermedad cíclica en México que, al igual que otras de este tipo, demande la capacitación continua del personal y presente incrementos en su frecuencia y en sus complicaciones.

La infección por ZIKV durante el embarazo debe sospecharse y diagnosticarse oportunamente, tanto en zonas endémicas como en zonas no endémicas, debido a la alta posibilidad de infección en mujeres embarazadas que se desplazan a las mismas, aunque sea de manera transitoria durante la gestación. De esta manera será posible ofrecer una atención integral a los pacientes, debido al alto riesgo de complicaciones que se pueden presentar. Aunque no todas las gestantes con ZIKV presentarán el síndrome congénito por Zika, se debe llevar un control prenatal mucho más estricto en las áreas endémicas. Este control deberá incluir la disponibilidad de pruebas confirmatorias, el fortalecimiento de la infraestructura de atención y una estrecha comunicación entre las áreas clínicas con el programa de control de vectores y la disminución de los riesgos de infección, para reducir la probabilidad de la picadura del mosquito transmisor del ZIKV en las poblaciones altamente vulnerables.

\section{Agradecimientos}

Los autores agradecen a la Dra. Sylvia Gabriela Cruz Núñez por la información proporcionada para la aclaración de la construcción del caso; a la Maestra en Ciencias Fabiola Reyes Román y la Maestra en Planeación y Administración de Servicios de Salud Pública. Jocelyne Led Jaime Rodríguez por sus comentarios en el proceso de elaboración del manuscrito y a las autoridades de los Servicios de Salud de Morelos por las facilidades otorgadas para la realización del mismo.

\section{Responsabilidades éticas}

Protección de personas y animales. Los autores declaran que para esta investigación no se han realizado experimentos en seres humanos ni en animales.

Confidencialidad de los datos. Los autores declaran que han seguido los protocolos de su centro de trabajo sobre la publicación de datos de pacientes.

Derecho a la privacidad y consentimiento informado. Los autores han obtenido el consentimiento informado de los pacientes o individuos referidos en el artículo. Este documento obra en poder del autor de correspondencia.

\section{Conflicto de intereses}

Los autores declaran no tener ningún conflicto de intereses. 


\section{Financiamiento}

Ninguno.

\section{Bibliografía}

1. Organización Panamericana de la Salud. Consideraciones provisionales para la atención de mujeres gestantes en escenarios con alta circulación del virus Zika: documento destinado a profesionales de Salud; 2016. Disponible en: http://iris.paho.org/xmlui/handle/123456789/28228.

2. Brasil P, Pereira JP Jr, Moreira ME, Ribeiro-Nogueira RM, Damasceno L, Wakimoto $\mathrm{M}$, et al. Zika virus infection in pregnant women in Rio de Janeiro. N Engl J Med. 2016;375:2321-34.

3. Pacheco O, Beltrán M, Nelson CA, Valencia D, Tolosa N, Farr SL, et al Zika virus disease in Colombia-preliminary report. $\mathrm{N}$ Engl J Med. 2016 Jun 15; [Epub ahead of print].

4. França GV, Schuler-Faccini L, Oliveira WK, Henriques CM, Carmo EH, Pedi VD, et al. Congenital Zika virus syndrome in Brazil: a case series of the first 1501 livebirths with complete investigation. Lancet. 2016;388:891-7.

5. Driggers RW, Ho CY, Korhonen EM, Kuivanen S, Jääskeläinen $A J$, Smura T, et al. Zika virus infection with prolonged maternal viremia and fetal brain abnormalities. N Engl J Med. 2016;374:2142-51.

6. Calvet G, Aguiar RS, Melo ASO, Sampaio SA, de Filippis I, Fabri A, et al. Detection and sequencing of Zika virus from amniotic fluid of fetuses with microcephaly in Brazil: a case study. Lancet Infect Dis. 2016;16:653-60.

7. Jurado KA, Simoni MK, Tang Z, Uraki R, Hwang J, Householder S, et al. Zika virus productively infects primary human placenta-specific macrophages. JCI Insight. 2016;1:e88461.

8. Bhatnagar Juli, Rabeneck DB, Martines RB, Reagan-Steiner S, Ermias Y, Estetter LB, et al. Zika virus RNA replication and persistence in brain and placental tissue. Emerg Infect Dis. 2017;23:405-14.

9. Contreras-Capetillo S, Valadez-González N, Manrique-Saide $P$, Carcaño-Castillo R, Pacheco-Tugores F, Barrera-Pérez $\mathrm{H}$, et al. Birth defects associated with congenital Zika virus infection in Mexico. Clin Pediatr (Phila). 2018;57:927-36.
10. Brasil-Martines R, Bhatnagar J, Oliveira-Ramos A, Freire-Davi $\mathrm{H}$, D'Andretta-Iglezias S, Takami-Kanamura C, et al. Pathology of congenital Zika syndrome in Brazil: a case series. Lancet. 2016;388:898-904.

11. Pan American Health Organization/World Health Organization. Zika resources: cases definitions; 2016. Disponible en: https://www.paho.org/hq/ index.php?option=com_content\&view=article\&id=11117\&Itemi$\mathrm{d}=41532$ \&lang=es.

12. Guerbois M, Fernández-Salas I, Azar SR, Danis-Lozano R, Alpuche-Aranda CM, Leal G, et al. Outbreak of Zika virus infection, Chiapas State, Mexico, 2015, and first confirmed transmission by Aedes aegypti mosquitoes in the Americas. J Infect Dis. 2016;214:1349-56.

13. Secretaría de Salud. Subsecretaría de Prevención y Promoción de la Salud. Dirección General de Epidemiología. Sistema de Vigilancia Epidemiológica de Enfermedad por virus del Zika. Casos confirmados de enfermedad por virus Zika. Semana epidemiológica 32 del 2018.

14. Secretaría de Salud. Subsecretaría de Prevención y Promoción de la Salud. Dirección General de Epidemiología. Sistema de Vigilancia Epidemiológica de Enfermedad por virus del Zika. Casos confirmados de síndrome congénito asociado a Zika, México 2016-2018. Disponible en: http://iris.paho.org/xmlui/bitstream/handle/123456789/49518/ 9789275320198 spa.pdf? sequence $=2 \&$ isAllowed $=y$

15. Loughna $P$, Chitty L, Evans T, Chudleigh T. Fetal size and dating: charts recommended for clinical obstetric practice. Brit Med Ultrasound Soc. 2009;17:161-7.

16. Petersen LR, Jamieson DJ, Powers AM, Honein MA. Zika virus. N Engl J Med. 2016;374:1552-63.

17. Rolland-Souza AS, Tenorio-Cordeiro M, Meneses JA, Honorato E, Araujo-Junior E, Da Silva-Castanha PM, et al. Clinical and laboratory diagnosis of congenital Zika virus syndrome and diaphragmatic unilateral palsy: case report. Rev Bras Saúde Mater Infant. 2016;16:467-73.

18. Mysorekar IU, Diamond MS. Modeling Zika virus infection in pregnancy. N Engl J Med. 2016;375:481-4

19. Martinot A, Abbink P, Afacan O, Prohl A, Bronson R, Hecht J, et al. Fetal neuropathology in Zika virus-infected pregnant female rhesus monkeys. Cell. 2018;173:1111-22.

20. Moore CA, Staples JE, Dobyns WB, Pessoa A, Ventura CV, Borges da Fonseca $\mathrm{E}$, et al. Characterizing the pattern of anomalies in congenital Zika syndrome for pediatric clinicians. JAMA Pediatr. 2017;171:288-95. 